\title{
К АЛГОРИТМИЗАЦИИ ОДНОГО МЕТОДА ШТРАФНЫХ ФУНКЦИИ ДЛЯ РЕШЕНИЯ ЗАДАЧИ ВЫПУКЛОГО ПРОГРАММИРОВАНИЯ
}

INGRID MAUER. OHE TRAHVIFUNKTSIOONIDE MEETODI ALGORITMEERIMISEST KUMERPROGRAMMEERIMISOLESANDE LAHENDAMISEKS

INGRID MAUER. ON ALGORITHMIZATION OF ONE METHOD OF PENALTY FUNCTIONS FOR SOLVING A CONVEX PROGRAMMING PROBLEM

Рассмотрим задачу выпуклого программирования

$$
\min \{f(x) \mid g(x) \leqslant 0\},
$$

где $x-n$-мерная точка евклидова пространства $E^{n}, f(x)$ и $g(x)^{\mathrm{T}}=$ $=\left(g_{1}(x), \ldots, g_{m}(x)\right)$ - функция и $m$-мерная вектор-функция соответственно, причем обе они непрерывно дифференцируемые и выпуклые, а область $\{x \mid g(x) \leqslant 0\}$ - непустая и ограниченная.

Среди методов решения этой задачи хорошо известен метод штрафных функций, суть которого заключается в последовательной безусловной минимизации функций

$$
F_{k}(x)=f(x)+\sum_{i=1}^{m} M_{i k}\left(g_{i}(x)_{+}\right)^{2},
$$

где

$$
g_{i}(x)_{+}=\max \left\{0, g_{i}(x)\right\} \quad(i=1, \ldots, m),
$$

а числа $M_{i k}>0$, причем последовательности $\left\{M_{i k}\right\}(i=1, \ldots, m)$ неубывающие и $M_{i k} \rightarrow \infty$ при $k \rightarrow \infty(i=1, \ldots, m)$ (см., напр., $\left.\left[{ }^{1,2}{ }^{2}\right]\right)$. При указанных выше предположениях метод сходится, т. е. расстоя ние от точки минимума функции $F_{k}(x)$ до множества всех решений задачи (1) сходится к нулю при $k \rightarrow \infty$ (это можно показать, используя, например, результаты из $\left.\left[{ }^{2}\right]\right)$.

В данном сообщении займемся проблемами алгоритмизации этого метода штрафных функций. Поскольку его вычислительные алгоритмы допускают лишь приближенное решение задачи (1), то аппроксимируем ее некоторой новой задачей и на основе последней, во-первых, выпишем вытекающие из нее условия заканчивания вычислений для этих алгоритмов и, во-вторых, обоснуем точность, которую в них при минимизации функций $F_{k}(x)$ повышать не обязательно.

Обозначим через $f_{x}\left(x^{0}\right)$ градиент функции $f(x)$ в точке $x^{0}$ и сформулируем вспомогательную задачу

$$
\min _{x}\left\{f(x)-F_{k x}(\bar{x})^{\mathrm{T}} x \mid g(x) \leqslant \varepsilon(\bar{x})\right\} .
$$


Здесь $\bar{x}$ - произвольно выбранная точка и $\varepsilon(x)^{\mathrm{T}}=\left(g_{1}(x)_{+}, \ldots\right.$, $\left.\ldots, g_{m}(x)_{+}\right)$. Так как точка $\bar{x}$ и неотрицательные числа $\bar{u}_{i}=$ $=2 M_{i k} g_{i}(\bar{x})_{+}(i=1, \ldots, m)$ такие, что

$$
\begin{gathered}
f_{x}(\bar{x})-F_{k x}(\bar{x})+\sum_{i=1}^{m} \bar{u}_{i} g_{i x}(\bar{x})=0, \\
\bar{u}_{i}\left(g_{i}(\bar{x})-g_{i}(\bar{x})_{+}\right)=0 \quad(i=1, \ldots, m), \\
g(\bar{x}) \leqslant \varepsilon(\bar{x}),
\end{gathered}
$$

то по $\left[{ }^{1}\right]$ (теорема 19) $\bar{x}$ есть решение задачи (2).

На основе задачи (2) аппроксимируем исходную задачу следующей задачей

$$
\begin{gathered}
\min _{x}\left\{f(x)-F_{k x}\left(x^{k}\right)^{\mathrm{T}} x \mid g(x) \leqslant \varepsilon\left(x^{k}\right) \leqslant \varepsilon,\right. \\
\left.\left\|F_{k x}\left(x^{k}\right)\right\| \leqslant \varepsilon_{0}\right\}^{*},
\end{gathered}
$$

где $\varepsilon^{\mathrm{T}}=\left(\varepsilon_{1}, \ldots, \varepsilon_{m}\right), \varepsilon_{i}(i=0,1, \ldots, m)$ - выбранные малые положительные числа, а функция $F_{k}(x)$ и точка $x^{k}$ - решение этой задачи. Отметим, что нормализацию вектора $F_{k x}\left(x^{k}\right)$ можно ввести и по-другому, например, отдельно по его компонентам.

Пусть задача (1) заменима задачей (3), т. е. любое решение задачи (3) содержит в качестве $x^{k}$ отыскиваемое решение задачи (1).

При этом предположении выпишем вытекающие из задачи (3) условия прекращения вычислений для алгоритмов описанного выше метода

$$
\begin{gathered}
\left\|F_{k x}(x)\right\| \leqslant \varepsilon_{0}, \\
g(x) \leqslant \varepsilon .
\end{gathered}
$$

В самом деле, если $F_{k}(x)$ и $x^{k}$, полученные в процессе вычислений, удовлетворяют условиям (4) и (5), то они являются решением задачи (3). так как $x^{k}$ есть решение задачи (2) при $\bar{x}=x^{k}$.

Минимизация функции $F_{k}(x)$ с точностью $\left\|F_{k x}(x)\right\| \leqslant c(c-$ положительное число) означает отыскание такой точки, которая удовлетворяет этому условию точности. Итак, условие (4) тоже определяет точность, которую в алгоритмах при минимизации функций $F_{k}(x)$ увеличивать не обязательно. Для доказательства этого утверждения обозначим через $x^{k}$ точку, которая удовлетворяет условию (4), и покажем, что $\varepsilon\left(x^{k}\right) \rightarrow 0$ при $k \rightarrow \infty$.

Действительно, выделим из последовательности $\left\{x^{k}\right\}$ сходящуюся подпоследовательность $\left\{x^{k_{\imath}}\right\}$, предельную точку которой обозначим через $x^{*}$. Это можно сделать, так как $\left\{x^{k}\right\}$ принадлежит к некоторому компактному множеству (доказательство здесь приводить не будем). Покажем, что $\varepsilon\left(x^{*}\right)=0$. Для этого введем точку-переменную $y \in E^{n}$ и обозначим

$$
L=\max _{y} \min _{x}\left\{f(x)-y^{\mathrm{T}} x \mid g(x) \leqslant 0, \quad\|y\| \leqslant \varepsilon_{0}\right\} .
$$

Если хоть при одном индексе $i$ функция $g_{i}\left(x^{*}\right)_{+}>0$, то для достаточно больших индексов $l$ получим

$$
F_{k_{t}}\left(x^{k_{t}}\right)-F_{k_{t} x}\left(x^{k_{t}}\right)^{\mathrm{T}} x^{k_{t}}>L,
$$

\footnotetext{
* Bсе нормы в тексте - нормы пространства $E^{n}$.
} 
что противоречит соотношению

$$
\begin{gathered}
F_{k_{l}}\left(x^{k_{l}}\right)-F_{k_{l} x}\left(x^{k_{l}}\right)^{\mathrm{T}} x^{k_{l}} \leqslant \min \left\{f(x)-F_{k_{l} x}\left(x^{k_{l}}\right)^{\mathrm{T}} x\right\} \\
g(x) \leqslant 0\} \leqslant L .
\end{gathered}
$$

Следовательно, $\varepsilon\left(x^{*}\right)=0$. Так как для любой сходящейся подпоследовательности $\left\{x^{k_{t}}\right\}$ из $\left\{x^{k}\right\}$ вектор $\varepsilon\left(x^{k_{t}}\right) \rightarrow 0$ при $l \rightarrow \infty$, то и $\varepsilon\left(x^{k}\right) \rightarrow 0$ при $k \rightarrow \infty$ (это нетрудно показать). Доказательство завершено.

Связывая полученные результаты с ранее известными результатами (см. $[3,4])$, предлагаем в алгоритмах рассматриваемого метода для решения задачи (1) минимизировать функцию $F_{k}(x)$ с точностью $\left\|F_{k x}(x)\right\| \leqslant \varepsilon^{k}$, где числа $\varepsilon^{k}>0$, причем $\varepsilon^{k} \rightarrow 0$ при $k \rightarrow \infty$, пока $\varepsilon^{k}>\varepsilon_{0}$, а затем с точностью (4) до выполнения условия (5) в полученной точке минимума. Для формирования функций $F_{k+1}(x)$ рекомендуем использовать приемы, изложенные в работах $\left[{ }^{2-5}\right]$.

\section{ЛИТЕ РАТ У РА}

1. Фи а к ко А., Мак-Кормик Г., Нелинейное программирование. Методы последовательной безусловной минимизации, М., 1972.

2. М а уэ р И. В., О методе штрафных функций, 17 с., Рукопись деп. в ВИНИТИ 27 марта 1975 г., № 849-75.

3. Костин а М. А., Тр. Ин-та матем. и мех. УНЦ АН СССР, вып. 14, Свердловск, 1974 , с. 65.

4. Поляк Б. Т., Ж. вычисл. матем. и матем. физики, 11, № 1, 3 (1971).

5. Ke efer D. L., Gottfried B. S., AIIE Trans., 2, No. 4, 281 (1970).

Ннститут кибернетики Академии наук Эстонской ССР
Поступила в редакцию 19/IV 1976 